\title{
A Chaotic System with an Infinite Number of Equilibrium Points: Dynamics, Horseshoe, and Synchronization
}

\author{
Viet-Thanh Pham, ${ }^{1}$ Christos Volos, ${ }^{2}$ Sundarapandian Vaidyanathan, ${ }^{3}$ and Xiong Wang ${ }^{4}$ \\ ${ }^{1}$ School of Electronics and Telecommunications, Hanoi University of Science and Technology, \\ 01 Dai Co Viet, Hanoi, Vietnam \\ ${ }^{2}$ Department of Physics, Aristotle University of Thessaloniki, 54124 Thessaloniki, Greece \\ ${ }^{3}$ Research and Development Centre, Vel Tech University, Avadi, Chennai 600062, India \\ ${ }^{4}$ Institute for Advanced Study, Shenzhen University, Shenzhen, Guangdong 518060, China \\ Correspondence should be addressed to Viet-Thanh Pham; pvt3010@gmail.com
}

Received 27 September 2016; Accepted 14 November 2016

Academic Editor: Zhi-Yuan Sun

Copyright (C) 2016 Viet-Thanh Pham et al. This is an open access article distributed under the Creative Commons Attribution License, which permits unrestricted use, distribution, and reproduction in any medium, provided the original work is properly cited.

\begin{abstract}
Discovering systems with hidden attractors is a challenging topic which has received considerable interest of the scientific community recently. This work introduces a new chaotic system having hidden chaotic attractors with an infinite number of equilibrium points. We have studied dynamical properties of such special system via equilibrium analysis, bifurcation diagram, and maximal Lyapunov exponents. In order to confirm the system's chaotic behavior, the findings of topological horseshoes for the system are presented. In addition, the possibility of synchronization of two new chaotic systems with infinite equilibria is investigated by using adaptive control.
\end{abstract}

\section{Introduction}

Nonlinear systems with chaotic behavior have been exploited since the 1960s [1-4]. Their applications have been witnessed in numerous areas, for example, secure digital communication systems [5], multiple input multiple output radar [6], image encryption with random bit sequence [7], or optimization algorithms [8]. Although almost normal chaotic systems have a countable number of equilibrium points, few unusual systems with infinite number of equilibria have been investigated in the last five years [9]. Chaotic system with line equilibrium was reported in [9-11]. A new class of chaotic systems with circle and square equilibrium was discovered by using predefined general forms $[12,13]$. In addition, hyperchaotic behavior was observed in a four-dimensional system with a curve of equilibria [14] or four-dimensional systems with a line of equilibria [15-17].

Remarkably, systems with an infinite number of equilibrium points are considered as systems with "hidden attractors" based on the view point of computation [18-21]. Hidden attractors cause unexpected effects for engineering systems [22-25]. However, the characteristics of hidden attractors are not well understood [26]. The community has raised some concerns about discovering hidden attractors in known systems $[27,28]$, finding new systems with hidden attractors $[29,30]$, studying synchronization schemes for systems with hidden attractors [31], or verifying chaotic dynamics in systems with hidden attractors with topological horseshoes [32, 33].

Motivated by special features of systems with hidden attractors, we introduce a new system with an open curve of equilibrium points in this work. In the next section, the model of the new system is described and its dynamics are discovered through different nonlinear tools. Chaotic dynamics of the proposed system are studied through topological horseshoes in Section 3. A possible synchronization of two new identical systems is discussed in Section 4. Finally, Section 5 concludes our work. 


\section{New System with an Infinite Number of Equilibrium Points and Its Properties}

The new system proposed in the present work is a threedimensional continuous system described as

$$
\begin{aligned}
& \dot{x}=-z, \\
& \dot{y}=x z^{2}, \\
& \dot{z}=x-y \tanh (y)+z\left(a y^{2}-z^{2}\right),
\end{aligned}
$$

in which three state variables are $x, y$, and $z$. It is worth noting that there is only one positive parameter $(a)$ in system (1).

It is straightforward to find the equilibrium points of the proposed system by setting the right hand side of (1) to equal zero, that is,

$$
\begin{aligned}
-z & =0, \\
x z^{2} & =0, \\
x-y \tanh (y)+z\left(a y^{2}-z^{2}\right) & =0 .
\end{aligned}
$$

Equation (2) reveals that $z=0$. By substituting $z=0$ into (3) and (4) we have

$$
x-y \tanh (y)=0
$$

In other words, system (1) has an infinite number of equilibrium points:

$$
\begin{aligned}
E & =\left\{(x, y, z) \in R^{3} \mid x=y^{*} \tanh \left(y^{*}\right), y=y^{*}, z\right. \\
& =0\} .
\end{aligned}
$$

For the equilibrium $E$, the Jacobian matrix of system (1) is given by

$$
\begin{aligned}
& \mathbf{J}_{E} \\
& =\left[\begin{array}{ccc}
0 & 0 & -1 \\
0 & 0 & 0 \\
1-\tanh \left(y^{*}\right)-y^{*}\left(1-\tanh ^{2}\left(y^{*}\right)\right) & a\left(y^{*}\right)^{2}
\end{array}\right] .
\end{aligned}
$$

On combining this result with $\operatorname{det}\left(\mathbf{J}_{E}-\lambda \mathbf{I}\right)=0$, we obtain its characteristic equation

$$
\lambda\left(\lambda^{2}-a\left(y^{*}\right)^{2} \lambda+1\right)=0 .
$$

It is easy to verify that the characteristic equation (8) has one zero eigenvalue $\left(\lambda_{1}=0\right)$ and two nonzero eigenvalues $\left(\lambda_{2,3}\right)$ which depend on the sign of the discriminant:

$$
\Delta=a^{2}\left(y^{*}\right)^{4}-4
$$

For $\Delta=0$, we get positive eigenvalues $\lambda_{2,3}=a\left(y^{*}\right)^{2} / 2$. Two nonzero eigenvalues are $\lambda_{2,3}=\left(a\left(y^{*}\right)^{2} \pm \sqrt{\Delta}\right) / 2$ for the positive discriminant. When the discriminant (9) is negative, a pair of complex conjugate eigenvalues is $\lambda_{2,3}=\left(a\left(y^{*}\right)^{2} \pm\right.$ $i \sqrt{\Delta}) / 2$. These eigenvalues state that the equilibrium point $E$ is unstable for $a>0$ and $y^{*} \neq 0$.

It is interesting that system (1) with uncountable equilibria is chaotic for $a=2.9$ and the initial condition $(x(0), y(0), z(0))=(0,0.1,0.2)$. Chaotic attractors of system (1) are presented in Figure 1. Its Lyapunov exponents and Kaplan-Yorke dimension are $L_{1}=0.0727, L_{2}=0, L_{3}=$ -0.3122 , and $D_{\mathrm{KY}}=2.2329$, respectively. The well-known Wolf's method [34] has been applied to calculate the Lyapunov exponents in our work. The time of computation is 10,000 . It is worth noted that, in general, in numerical experiments one cannot expect to get the same values of the finitetime local Lyapunov exponents and the Lyapunov dimension for different points [35-37]. Therefore, the maximum of the finite-time local Lyapunov dimensions on the grid of points has to be considered [35-37].

The value of parameter $a$ has been changed to get detailed dynamics of system (1) with infinite equilibria. By decreasing the value of the parameter $a$ from 3.4 to 2.8 , the bifurcation diagram and maximal Lyapunov exponents (MLEs) of system (1) are shown in Figures 2 and 3, respectively. It is possible to observe a route from period-doubling limit cycles to chaos when decreasing the value of the parameter $a$. When $a>$ 3.048, system (1) remains at periodical states, for example, periodical states for $a=3.35$ are illustrated in Figure 4 . System (1) can generate chaotic attractors for $a \leq 3.048$.

\section{Horseshoe in the Chaotic System with Infinite Equilibria}

Topological horseshoe is a different effective approach to investigate chaos in dynamical systems [38-44]. There is significant attention about seeking topological horseshoe in chaotic systems with hidden attractors $[32,33]$. Therefore, in this section we will discover topological horseshoes in the proposed system with infinite equilibria (1).

In order to support the verification of chaos in system with infinite equilibria (1), the most important results of topological horseshoe [45-47] are reviewed briefly. We define $X$ and $D$ as a metric space and a compact subset while $f$ is a map $f: D \rightarrow X$. We assume that there are $m$ mutually disjoint compact subsets of $D$ (i.e., $D_{1}, D_{2}, \ldots, D_{m}$ ) and the restriction of $f$ to each $D_{i}$ is continuous. A compact subset $d$ of $D$ satisfies $d_{i}=d \cap D_{i}$ for $1 \leq i \leq m$. In this case, $d$ is a connection with respect to $m$ mutually disjoint compact subsets of $D$. We denote $F$ as a family of connections with respect to $m$ mutually disjoint compact subsets of $D$. The family $F$ is an $f$-connected family with respect to $m$ mutually disjoint compact subsets of $D$ when

$$
\begin{array}{r}
d \in F \Longrightarrow \\
f\left(d_{i}\right) \in F .
\end{array}
$$

Horseshoe Lemma (see [48]). If there is an f-connected family $F$ with respect to $m$ mutually disjoint compact subsets of $D$, then there is the presence of a compact invariant set $K \subset D$ and semiconjugate to $m$-shift dynamics is $f \mid K$. 


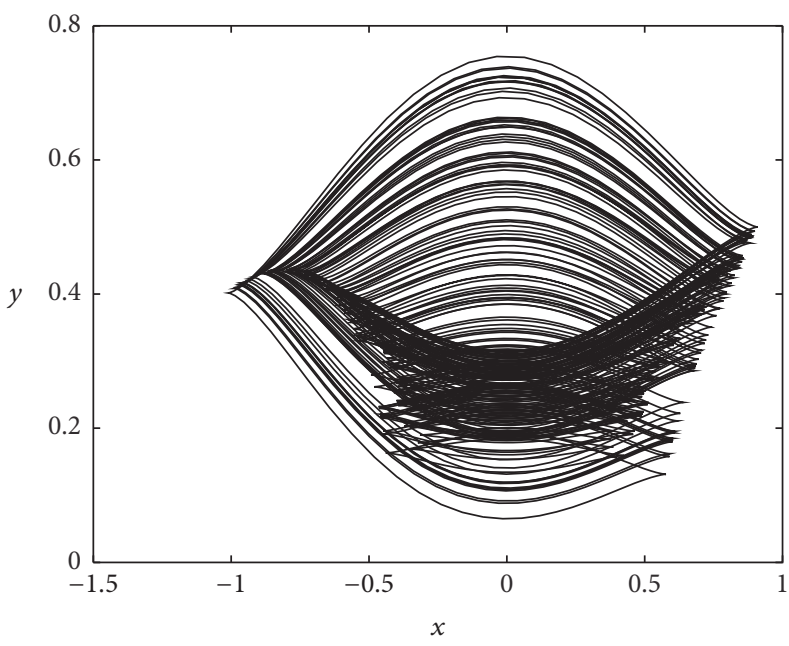

(a)

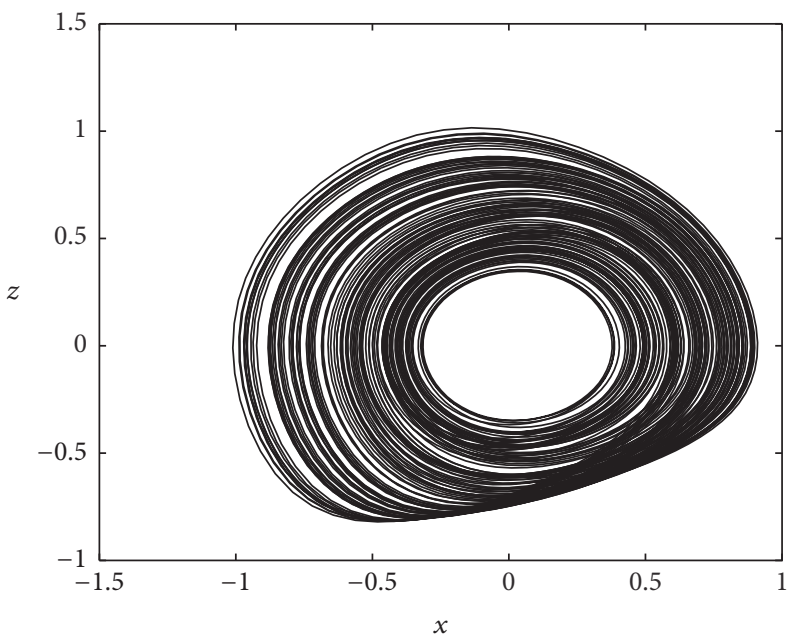

(b)

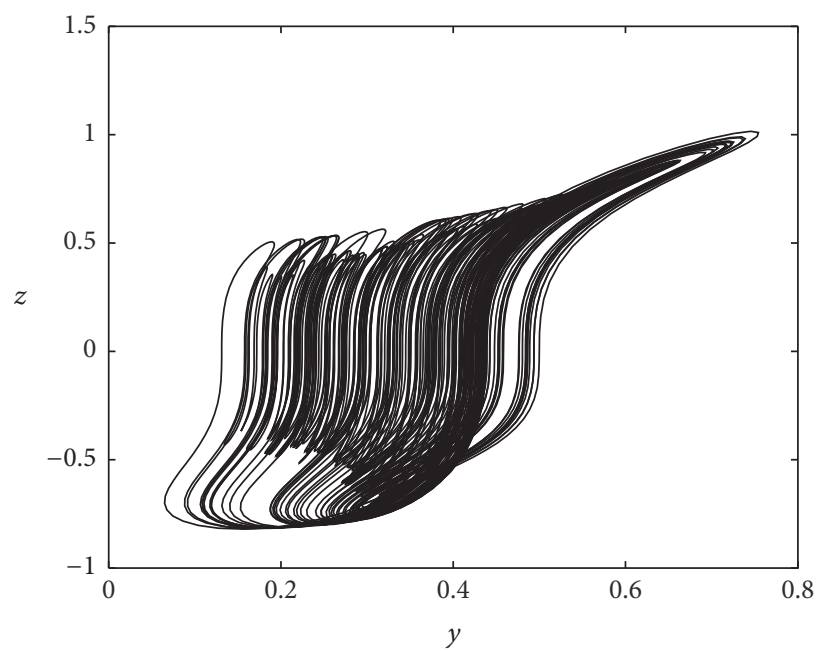

(c)

FIGURE 1: Chaotic attractor of the system with infinite equilibria (1) in (a) $x-y$ plane, (b) $x-z$ plane, and (c) $y-z$ plane.

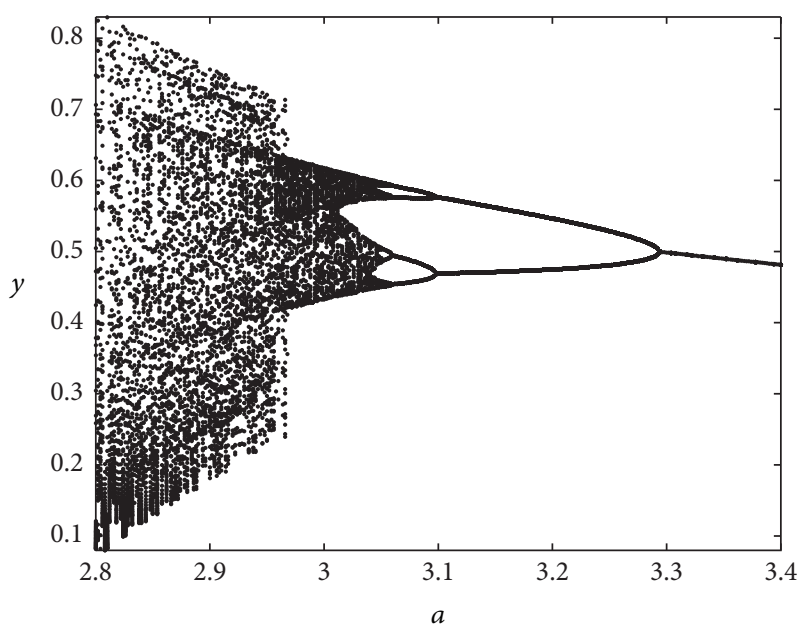

FIGURE 2: Bifurcation diagram of the system with infinite equilibria (1) when varying $a$.

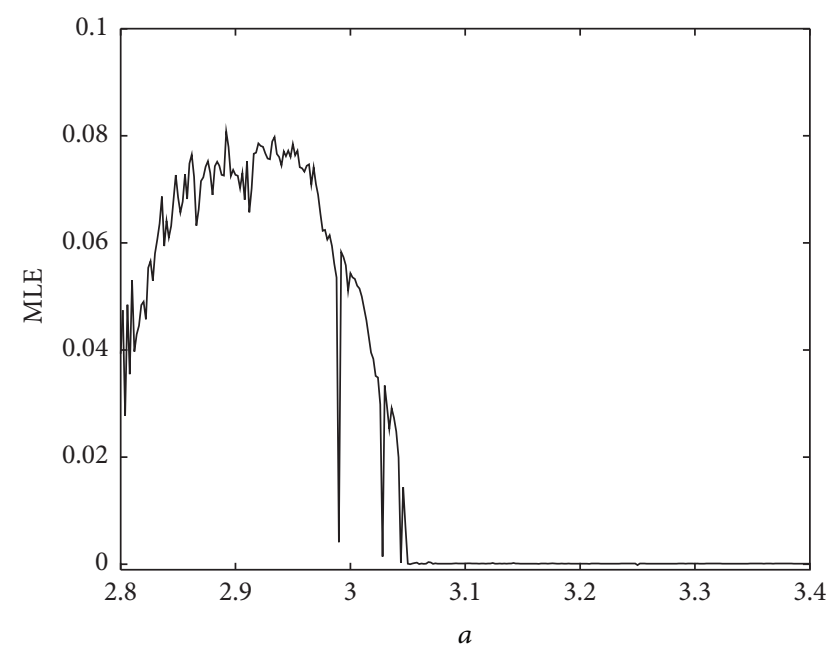

FIGURE 3: Maximal Lyapunov exponents of system (1) with respect to the bifurcation parameter $a$. 


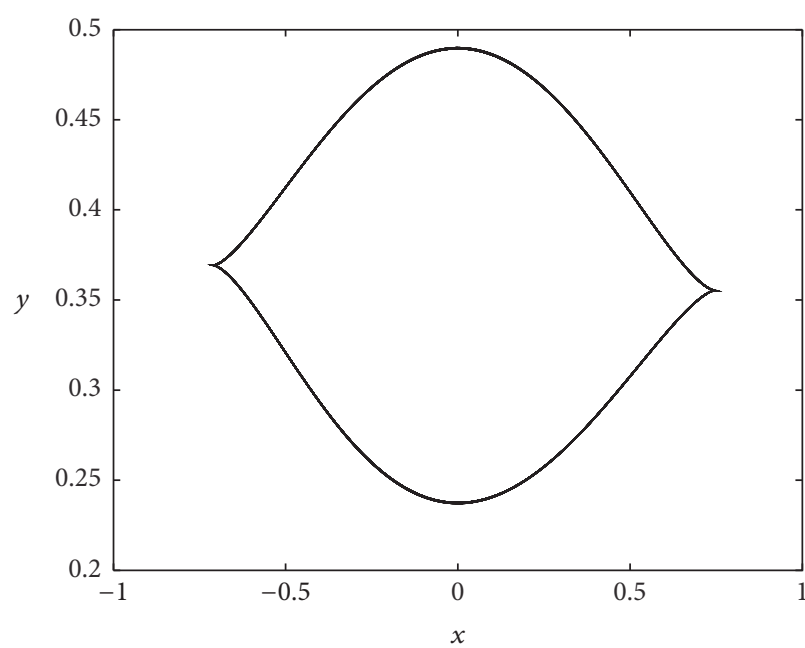

(a)

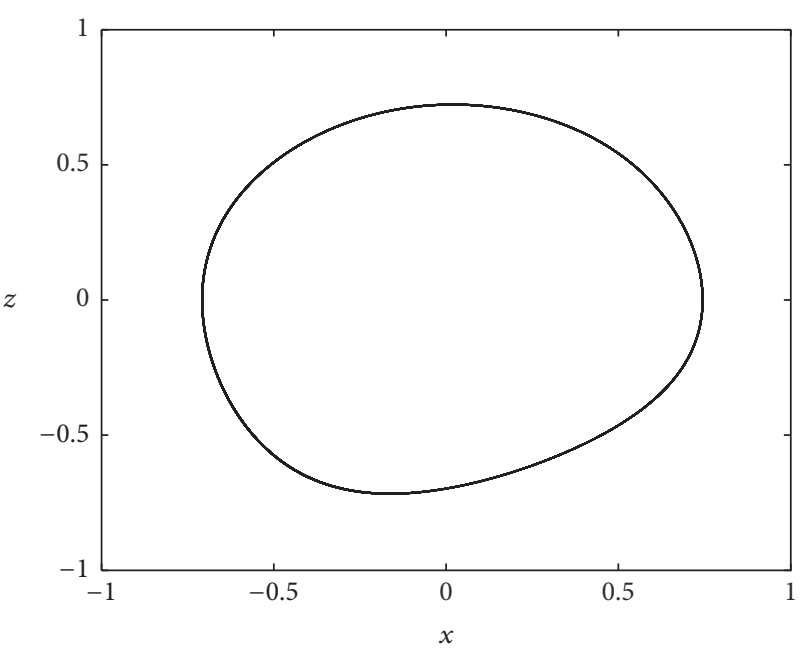

(b)

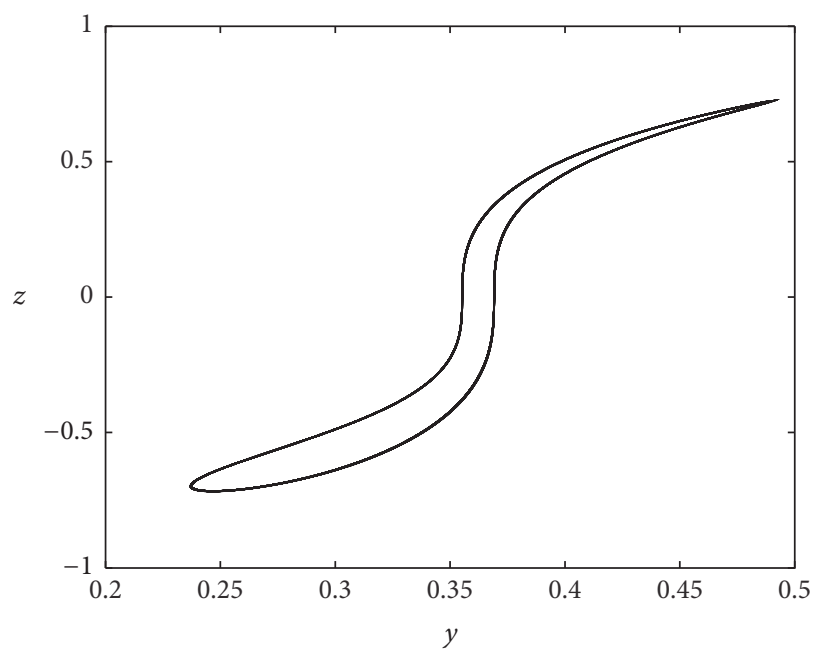

(c)

FIgURE 4: Limit cycle of the system with infinite equilibria (1) in (a) $x-y$ plane, (b) $x-z$ plane, and (c) $y-z$ plane for $a=3.35$.

In order to find the topology horseshoe, we select two polygon subsets $D_{1}, D_{2}$ in the Poincaré map $\Gamma$ of the system with infinite equilibria (1):

$$
\Gamma=\left\{(x, y, z) \in R^{3} \mid z=0\right\} .
$$

The corresponding Poincaré map $H$ is defined as

$$
H: \Gamma \longrightarrow \Gamma \text {. }
$$

Here $H(p)$ is the image of the initial $p$ that returns back to $\Gamma$ at the first time [48]. The same definition can be applied to the corresponding Poincaré map $H^{n}$. In this work, four vertices of the first polygon subset $D_{1}$ are selected as

$$
\begin{aligned}
& (0.822470322,0.438565370), \\
& (0.823776275,0.435278699), \\
& (0.815679371,0.429691358), \\
& (0.813328658,0.432978029),
\end{aligned}
$$

while four vertices of the second polygon subset $D_{2}$ are chosen as

$$
\text { (0.796873661, 0.424104017), }
$$$$
\text { (0.798701994, 0.421146013), }
$$$$
\text { (0.794261757, 0.418188009), }
$$

$$
(0.792694615,0.420160011) \text {. }
$$

Two selected polygon subsets and their images are displayed in Figures 5 and 6. As shown in Figure 5, it is trivial to verify that $H^{6}\left(D_{1}\right)$ goes through both two polygon subsets $D_{1}$ and $D_{2}$. Similarly, $H^{6}\left(D_{2}\right)$ crosses two polygon subsets $D_{1}$ and $D_{2}$ as illustrated in Figure 6. According to the Horseshoe lemma, chaos of the system with infinite equilibria (1) is determined [45-47]. 


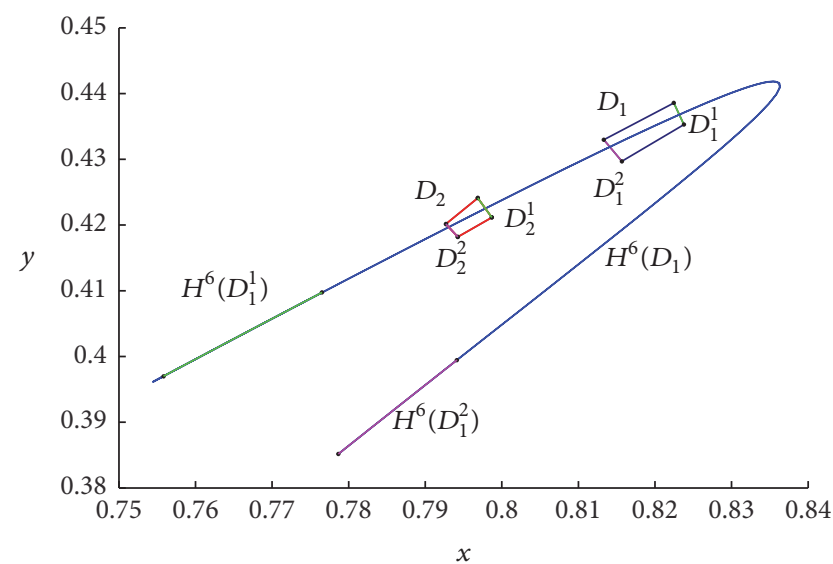

Figure 5: The quadrilateral subset $D_{1}$ and its image.

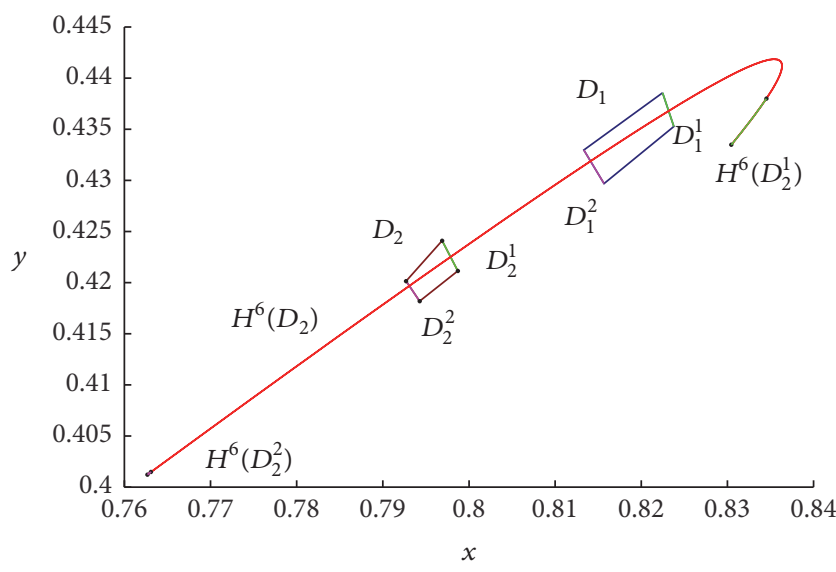

FIgURE 6: The quadrilateral subset $D_{2}$ and its image.

\section{Synchronization of the Identical Systems with Infinite Equilibria}

After the study of Pecora and Carroll about synchronization in chaotic systems [49], various synchronization techniques and related works were presented extensively [50-54]. Critically, the possibility of synchronization of two identical chaotic systems plays a vital role in practical applications [5558]. In this section, we discover the synchronization of two new systems with infinite equilibria, called the master system and the slave system, by using an adaptive controller.

We consider the following master system with the unknown system parameter $a$ :

$$
\begin{aligned}
& \dot{x}_{1}=-z_{1}, \\
& \dot{y}_{1}=x_{1} z_{1}^{2}, \\
& \dot{z}_{1}=x_{1}-y_{1} \tanh \left(y_{1}\right)+a y_{1}^{2} z_{1}-z_{1}^{3} .
\end{aligned}
$$

The slave system with adaptive control $\mathbf{u}=\left[u_{x}, u_{y}, u_{z}\right]^{T}$ is given as

$$
\begin{aligned}
& \dot{x}_{2}=-z_{2}+u_{x}, \\
& \dot{y}_{2}=x_{2} z_{2}^{2}+u_{y}, \\
& \dot{z}_{2}=x_{2}-y_{2} \tanh \left(y_{2}\right)+a y_{2}^{2} z_{2}-z_{2}^{3}+u_{z} .
\end{aligned}
$$

The state errors between the slave system and the master system are calculated by

$$
\begin{aligned}
& e_{x}=x_{2}-x_{1}, \\
& e_{y}=y_{2}-y_{1}, \\
& e_{z}=z_{2}-z_{1} .
\end{aligned}
$$

The parameter estimation error is defined as follows

$$
e_{a}=a-\widehat{a},
$$

in which $\hat{a}$ is the estimation of the unknown parameter $a$.

In order to synchronize the slave system and the master system, the adaptive control is constructed in the following form:

$$
\begin{aligned}
u_{x}= & e_{z}-k_{x} e_{x}, \\
u_{y}= & -x_{2} z_{2}^{2}+x_{1} z_{1}^{2}-k_{y} e_{y}, \\
u_{z}= & -e_{x}+y_{2} \tanh \left(y_{2}\right)-y_{1} \tanh \left(y_{1}\right) \\
& -\hat{a}\left(y_{2}^{2} z_{2}-y_{1}^{2} z_{1}\right)+z_{2}^{3}-z_{1}^{3}-k_{z} e_{z},
\end{aligned}
$$

in which $k_{x}, k_{y}$, and $k_{z}$ are three positive gain constants and the parameter update law is described by

$$
\dot{\hat{a}}=e_{z}\left(y_{2}^{2} z_{2}-y_{1}^{2} z_{1}\right)
$$

By applying Lyapunov stability theory, we will prove that the master system (15) and the slave system (16) are synchronized when using the adaptive control (19).

In this work, the Lyapunov function is selected as

$$
V\left(e_{x}, e_{y}, e_{z}, e_{a}\right)=\frac{1}{2}\left(e_{x}^{2}+e_{y}^{2}+e_{z}^{2}+e_{a}^{2}\right)
$$

Therefore, the differentiation of $V$ is

$$
\dot{V}=e_{x} \dot{e}_{x}+e_{y} \dot{e}_{y}+e_{z} \dot{e}_{z}+e_{a} \dot{e}_{a} .
$$

From (17) and (18), we have

$$
\begin{aligned}
& \dot{e}_{x}=-k_{x} e_{x}, \\
& \dot{e}_{y}=-k_{y} e_{y}, \\
& \dot{e}_{z}=e_{a}\left(y_{2}^{2} z_{2}-y_{1}^{2} z_{1}\right)-k_{z} e_{z}, \\
& \dot{e}_{a}=-\dot{\hat{a}} .
\end{aligned}
$$


By substituting (23) into (22), the differentiation of $V$ can be expressed as

$$
\dot{V}=-k_{x} e_{x}^{2}-k_{y} e_{y}^{2}-k_{z} e_{z}^{2}
$$

Because $\dot{V}$ is a negative semidefinite function, it is simply verified that $e_{x} \rightarrow 0, e_{y} \rightarrow 0$, and $e_{z} \rightarrow 0$ exponentially as $t \rightarrow \infty$ according to Barbalat's lemma [59]. In other words, we obtain the complete synchronization between the master system and the slave system.

We take an example to illustrate the calculation of the synchronization scheme. The parameter values of the master system and the slave system are fixed as

$$
a=2.9 \text {. }
$$

We assume that the initial states of the master system are

$$
\begin{aligned}
& x_{1}(0)=0, \\
& y_{1}(0)=0.1, \\
& z_{1}(0)=0.2,
\end{aligned}
$$

while the initial states of the slave system are

$$
\begin{aligned}
& x_{2}(0)=-0.7 \\
& y_{2}(0)=0.4 \\
& z_{2}(0)=-0.1 .
\end{aligned}
$$

The positive gain constants are chosen as follows: $k_{x}=4, k_{y}=$ 4 , and $k_{z}=4$. We take the initial condition of the parameter estimate as

$$
\widehat{a}(0)=3 \text {. }
$$

The time-history of the synchronization errors $e_{x}, e_{y}, e_{z}$ is shown in Figure 7. It is straightforward to verify that Figure 7 depicts the synchronization of the master and slave systems.

\section{Conclusions}

A new chaotic system with a curve of equilibria has been introduced in this work. Interestingly, because of having an infinite number of equilibrium points, the system is a special system with hidden attractors, which is rarely reported in the literature. Basic dynamical characters of the system with infinite equilibria are investigated via phase portraits, equilibrium analysis, Kaplan-Yorke dimension, maximal Lyapunov exponents, and bifurcation diagram. Although it is great challenge for researchers to find a topological horseshoe in systems with hidden attractor, horseshoe in such new system with infinite equilibria has been discovered in our work. After studying the possibility of synchronization of two novel chaotic systems, we believe that potential applications of such a system should be considered further in future works.

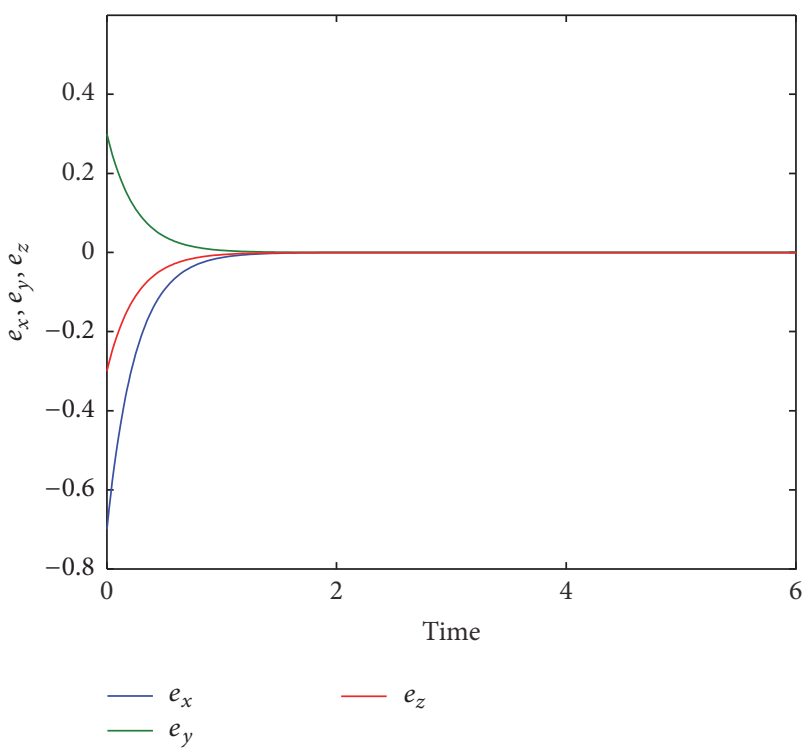

FIGURE 7: Time-history of the synchronization errors which indicates the synchronization between the master system and the slave system.

\section{Competing Interests}

The authors declare that there is no conflict of interests regarding the publication of this paper.

\section{Acknowledgments}

The authors are grateful to Professor Qingdu Li, Research Center of Analysis and Control for Complex Systems, Chongqing University of Post and Telecommunication, China, for his useful discussions and suggestions. The author Xiong Wang was supported by the National Natural Science Foundation of China (no. 61601306) and Shenzhen Overseas High Level Talent Peacock Project Fund (no. 20150215145C).

\section{References}

[1] E. N. Lorenz, "Deterministic nonperiodic flow," Journal of the Atmospheric Sciences, vol. 20, pp. 130-141, 1963.

[2] S. H. Strogatz, Nonlinear Dynamics and Chaos: With Applications to Physics, Biology, Chemistry, and Engineering, Perseus Books, Massachusetts, Mass, USA, 1994.

[3] S. Vaidyanathan and S. Rasappan, "Global Chaos Synchronization of $n$-Scroll Chua Circuit and Lur'e System using Backstepping Control Design with Recursive Feedback," Arabian Journal for Science and Engineering, vol. 39, no. 4, pp. 3351-3364, 2014.

[4] J. Sun, Y. Shen, and G. Cui, "Compound synchronization of four chaotic complex systems," Advances in Mathematical Physics, vol. 2015, Article ID 921515, 11 pages, 2015.

[5] Y.-Y. Hou, H.-C. Chen, J.-F. Chang, J.-J. Yan, and T.-L. Liao, "Design and implementation of the Sprott chaotic secure digital communication systems," Applied Mathematics and Computation, vol. 218, no. 24, pp. 11799-11805, 2012.

[6] M. Yang and G. Zhang, "An efficient method for anti-jamming in CS-MIMO chaotic radar direction finding," Arabian Journal for Science and Engineering, vol. 39, no. 12, pp. 8947-8955, 2014. 
[7] H. Khanzadi, M. Eshghi, and S. E. Borujeni, "Image encryption using random bit sequence based on chaotic maps," Arabian Journal for Science and Engineering, vol. 39, no. 2, pp. 1039-1047, 2014.

[8] K. Tatsumi, T. Ibuki, and T. Tanino, "A chaotic particle swarm optimization exploiting a virtual quartic objective function based on the personal and global best solutions," Applied Mathematics and Computation, vol. 219, no. 17, pp. 8991-9011, 2013.

[9] S. Jafari and J. C. Sprott, "Simple chaotic flows with a line equilibrium," Chaos, Solitons \& Fractals, vol. 57, pp. 79-84, 2013.

[10] C. Li and J. C. Sprott, "Chaotic flows with a single nonquadratic term," Physics Letters. A, vol. 378, no. 3, pp. 178-183, 2014.

[11] C. Li, J. C. Sprott, Z. Yuan, and H. Li, "Constructing chaotic systems with total amplitude control," International Journal of Bifurcation and Chaos in Applied Sciences and Engineering, vol. 25, no. 10, Article ID 1530025, 14 pages, 2015.

[12] T. Gotthans and J. Petržela, "New class of chaotic systems with circular equilibrium," Nonlinear Dynamics, vol. 81, no. 3, pp. 1143-1149, 2015.

[13] T. Gotthans, J. C. Sprott, and J. Petrzela, "Simple chaotic flow with circle and square equilibrium," International Journal of Bifurcation and Chaos in Applied Sciences and Engineering, vol. 26, no. 8, Article ID 1650137, 8 pages, 2016.

[14] Y. Chen and Q. Yang, "A new Lorenz-type hyperchaotic system with a curve of equilibria," Mathematics and Computers in Simulation, vol. 112, pp. 40-55, 2015.

[15] C. Li, J. C. Sprott, and W. Thio, "Bistability in a hyperchaotic system with a line equilibrium," Journal of Experimental and Theoretical Physics, vol. 118, no. 3, pp. 494-500, 2014.

[16] Q. Li, S. Tang, H. Zeng, and T. Zhou, "On hyperchaos in a small memristive neural network," Nonlinear Dynamics, vol. 78, no. 2, pp. 1087-1099, 2014.

[17] Q. Li, H. Zeng, and J. Li, "Hyperchaos in a 4D memristive circuit with infinitely many stable equilibria," Nonlinear Dynamics, vol. 79, no. 4, pp. 2295-2308, 2015.

[18] G. A. Leonov, N. V. Kuznetsov, O. A. Kuznetsova, S. M. Seledzhi, and V. I. Vagaitsev, "Hidden oscillations in dynamical systems," Transactions on Systems and Control, vol. 6, no. 2, pp. 54-67, 2011.

[19] G. A. Leonov, N. V. Kuznetsov, and V. I. Vagaitsev, "Localization of hidden Chua's attractors," Physics Letters. A, vol. 375, no. 23, pp. 2230-2233, 2011.

[20] G. A. Leonov, N. V. Kuznetsov, and V. I. Vagaitsev, "Hidden attractor in smooth Chua systems," Physica D. Nonlinear Phenomena, vol. 241, no. 18, pp. 1482-1486, 2012.

[21] G. A. Leonov and N. V. Kuznetsov, "Hidden attractors in dynamical systems. From hidden oscillations in Hilbert-Kolmogorov, Aizerman, and KALman problems to hidden chaotic attractor in Chua circuits," International Journal of Bifurcation and Chaos in Applied Sciences and Engineering, vol. 23, no. 1, Article ID 1330002, 69 pages, 2013.

[22] G. A. Leonov, N. V. Kuznetsov, M. A. Kiseleva, E. P. Solovyeva, and A. M. Zaretskiy, "Hidden oscillations in mathematical model of drilling system actuated by induction motor with a wound rotor," Nonlinear Dynamics, vol. 77, no. 1-2, pp. 277-288, 2014.

[23] A. Chudzik, P. Perlikowski, A. Stefanski, and T. Kapitaniak, "Multistability and rare attractors in van der Pol-duffing oscillator," International Journal of Bifurcation and Chaos in Applied Sciences and Engineering, vol. 21, no. 7, pp. 1907-1912, 2011.
[24] Z. T. Zhusubaliyev and E. Mosekilde, "Multistability and hidden attractors in a multilevel DC/DC converter," Mathematics and Computers in Simulation, vol. 109, pp. 32-45, 2015.

[25] G. A. Leonov, N. V. Kuznetsov, and T. N. Mokaev, "Hidden attractor and homoclinic orbit in Lorenz-like system describing convective fluid motion in rotating cavity," Communications in Nonlinear Science and Numerical Simulation, vol. 28, no. 1-3, pp. 166-174, 2015.

[26] D. Dudkowski, S. Jafari, T. Kapitaniak, N. V. Kuznetsov, G. A. Leonov, and A. Prasad, "Hidden attractors in dynamical systems," Physics Reports. A Review Section of Physics Letters, vol. 637, pp. 1-50, 2016.

[27] S. Brezetskyi, D. Dudkowski, and T. Kapitaniak, "Rare and hidden attractors in Van der Pol-Duffing oscillators," European Physical Journal: Special Topics, vol. 224, no. 8, pp. 1459-1467, 2015.

[28] Z. T. Zhusubaliyev, E. Mosekilde, V. G. Rubanov, and R. A. Nabokov, "Multistability and hidden attractors in a relay system with hysteresis," Physica D. Nonlinear Phenomena, vol. 306, pp. 6-15, 2015.

[29] Z. Wei, R. Wang, and A. Liu, "A new finding of the existence of hidden hyperchaotic attractors with no equilibria," Mathematics and Computers in Simulation, vol. 100, pp. 13-23, 2014.

[30] S. Jafari, J. C. Sprott, and F. Nazarimehr, "Recent new examples of hidden attractors," European Physical Journal: Special Topics, vol. 224, no. 8, pp. 1469-1476, 2015.

[31] Y. Feng, J. Pu, and Z. Wei, "Switched generalized function projective synchronization of two hyperchaotic systems with hidden attractors," European Physical Journal: Special Topics, vol. 224, no. 8, pp. 1593-1604, 2015.

[32] P. Zhou and F. Yang, "Hyperchaos, chaos, and horseshoe in a 4D nonlinear system with an infinite number of equilibrium points," Nonlinear Dynamics, vol. 76, no. 1, pp. 473-480, 2014.

[33] Q. Li, S. Hu, S. Tang, and G. Zeng, "Hyperchaos and horseshoe in a $4 \mathrm{D}$ memristive system with a line of equilibria and its implementation," International Journal of Circuit Theory and Applications, vol. 42, no. 11, pp. 1172-1188, 2014.

[34] A. Wolf, J. B. Swift, H. L. Swinney, and J. A. Vastano, "Determining Lyapunov exponents from a time series," Physica D: Nonlinear Phenomena, vol. 16, no. 3, pp. 285-317, 1985.

[35] N. V. Kuznetsov, "The Lyapunov dimension and its estimation via the Leonov method," Physics Letters. A, vol. 380, no. 25-26, pp. 2142-2149, 2016.

[36] N. V. Kuznetsov, T. A. Alexeeva, and G. A. Leonov, "Invariance of Lyapunov exponents and Lyapunov dimension for regular and irregular linearizations," Nonlinear Dynamics. An International Journal of Nonlinear Dynamics and Chaos in Engineering Systems, vol. 85, no. 1, pp. 195-201, 2016.

[37] G. A. Leonov, N. V. Kuznetsov, N. A. Korzhemanova, and D. V. Kusakin, "Lyapunov dimension formula for the global attractor of the Lorenz system," Communications in Nonlinear Science and Numerical Simulation, vol. 41, pp. 84-103, 2016.

[38] Q. Li, "A topological horseshoe in the hyperchaotic Rossler attractor," Physics Letters. A, vol. 372, no. 17, pp. 2989-2994, 2008.

[39] Q. Li and X.-S. Yang, "Two kinds of horseshoes in a hyperchaotic neural network," International Journal of Bifurcation and Chaos, vol. 22, no. 8, Article ID 1250200, 2012.

[40] K. Deng and S. Yu, "Estimating ultimate bound and finding topological horseshoe for a new chaotic system," Optik, vol. 125, no. 20, pp. 6044-6048, 2014. 
[41] J. He, S. Yu, and J. Cai, "Topological horseshoe analysis for a three-dimensional anti-control system and its application," Optik-International Journal for Light and Electron Optics, vol. 127, no. 20, pp. 9444-9456, 2016.

[42] Z. Liao and Y. Huang, "Horseshoe and topological entropy estimate of a class of three-dimensional cellular neural networks," Applied Mathematics and Computation, vol. 197, no. 1, pp. 382388, 2008.

[43] Q.-J. Fan, "Horseshoe in a modified Van der Pol-Duffing circuit," Applied Mathematics and Computation, vol. 210, no. 2, pp. 436-440, 2009.

[44] X.-S. Yang, Q. Li, and S. Cheng, "Horseshoe chaos and topological entropy estimate in a simple power system," Applied Mathematics and Computation, vol. 211, no. 2, pp. 467-473, 2009.

[45] X.-S. Yang, "Topological horseshoes in continuous maps," Chaos, Solitons and Fractals, vol. 33, no. 1, pp. 225-233, 2007.

[46] Q. Li and X.-S. Yang, "A simple method for finding topological horseshoes," International Journal of Bifurcation and Chaos in Applied Sciences and Engineering, vol. 20, no. 2, pp. 467-478, 2010.

[47] Q. Li, X.-S. Yang, and S. Chen, "Hyperchaos in a spacecraft power system," International Journal of Bifurcation and Chaos, vol. 21, no. 6, pp. 1719-1726, 2011.

[48] X.-S. Yang, "Topological horseshoes and computer assisted verification of chaotic dynamics," International Journal of Bifurcation and Chaos, vol. 19, no. 4, pp. 1127-1145, 2009.

[49] L. M. Pecora and T. L. Carroll, "Synchronization in chaotic systems," Physical Review Letters, vol. 64, no. 8, pp. 821-824, 1990.

[50] M.-C. Pai, "Global synchronization of uncertain chaotic systems via discrete-time sliding mode control," Applied Mathematics and Computation, vol. 227, pp. 663-671, 2014.

[51] V. Sundarapandian and I. Pehlivan, "Analysis, control, synchronization, and circuit design of a novel chaotic system," Mathematical and Computer Modelling, vol. 55, no. 7-8, pp. 1904-1915, 2012.

[52] X. Zhao, Z. Li, and S. Li, "Synchronization of a chaotic finance system," Applied Mathematics and Computation, vol. 217, no. 13, pp. 6031-6039, 2011.

[53] S. C. Jeong, D. H. Ji, J. H. Park, and S. C. Won, "Adaptive synchronization for uncertain chaotic neural networks with mixed time delays using fuzzy disturbance observer," Applied Mathematics and Computation, vol. 219, no. 11, pp. 5984-5995, 2013.

[54] R. Martínez-Guerra, D. M. Corona-Fortunio, and J. L. MataMachuca, "Synchronization of chaotic Liouvillian systems: an application to Chua's oscillator," Applied Mathematics and Computation, vol. 219, no. 23, pp. 10934-10944, 2013.

[55] S. Boccaletti, J. Kurths, G. Osipov, D. L. Valladares, and C. S. Zhou, "The synchronization of chaotic systems," Physics Reports. A Review Section of Physics Letters, vol. 366, no. 1-2, pp. 1-101, 2002.

[56] L. Fortuna and M. Frasca, "Experimental synchronization of single-transistor-based chaotic circuits," Chaos, vol. 17, no. 4, Article ID 043118, 2007.

[57] A. Abdullah, "Synchronization and secure communication of uncertain chaotic systems based on full-order and reducedorder output-affine observers," Applied Mathematics and Computation, vol. 219, no. 19, pp. 10000-10011, 2013.

[58] J. Sun, J. Guo, C. Yang, A. Zheng, and X. Zhang, "Adaptive generalized hybrid function projective dislocated synchronization of new four-dimensional uncertain chaotic systems," Applied Mathematics and Computation, vol. 252, pp. 304-314, 2015.

[59] H. K. Khalil, Nonlinear Systems, Prentice Hall, New Jersey, NJ, USA, 3rd edition, 2002. 


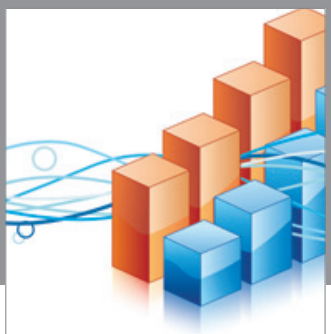

Advances in

Operations Research

vatem alat4

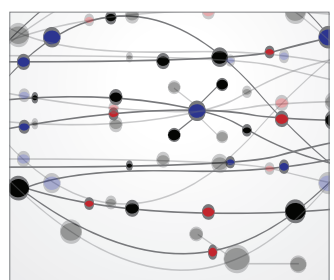

\section{The Scientific} World Journal
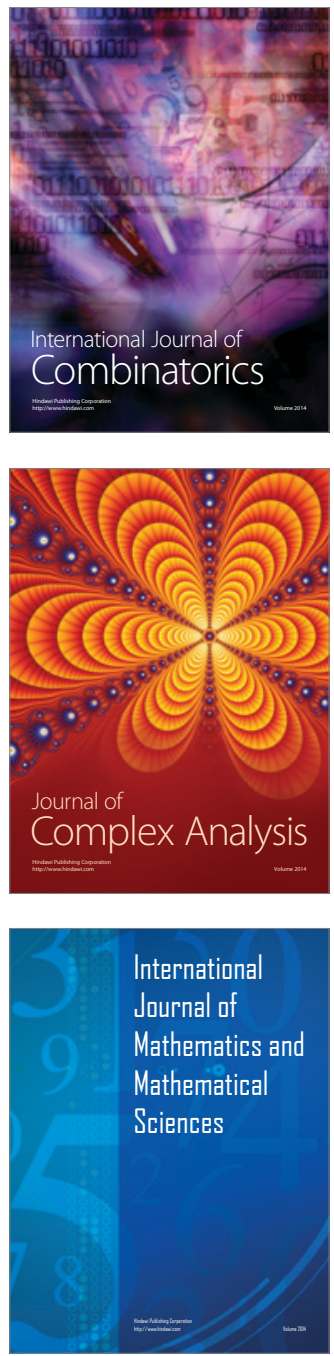
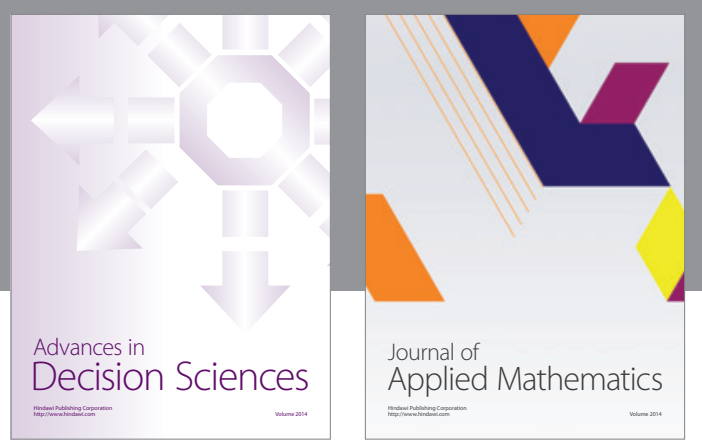

Algebra

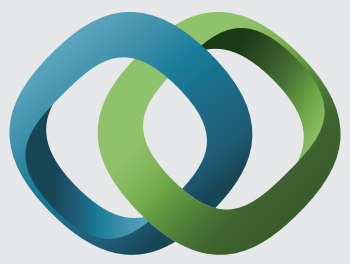

\section{Hindawi}

Submit your manuscripts at

http://www.hindawi.com
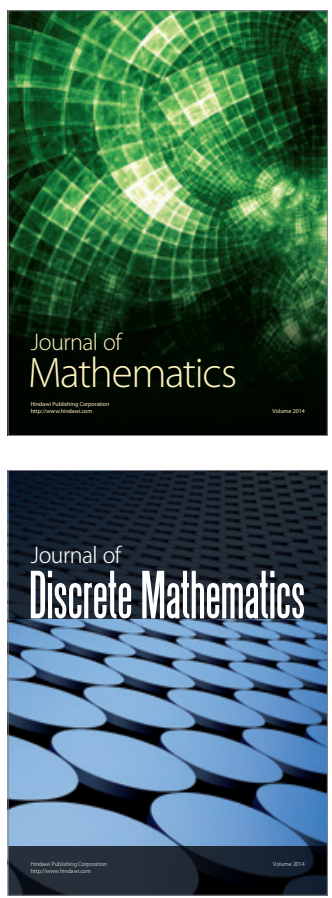

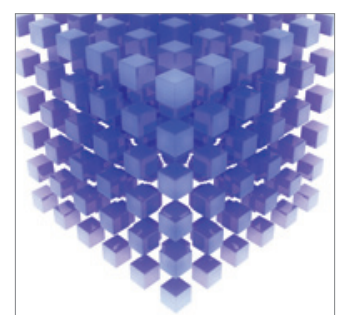

Mathematical Problems in Engineering
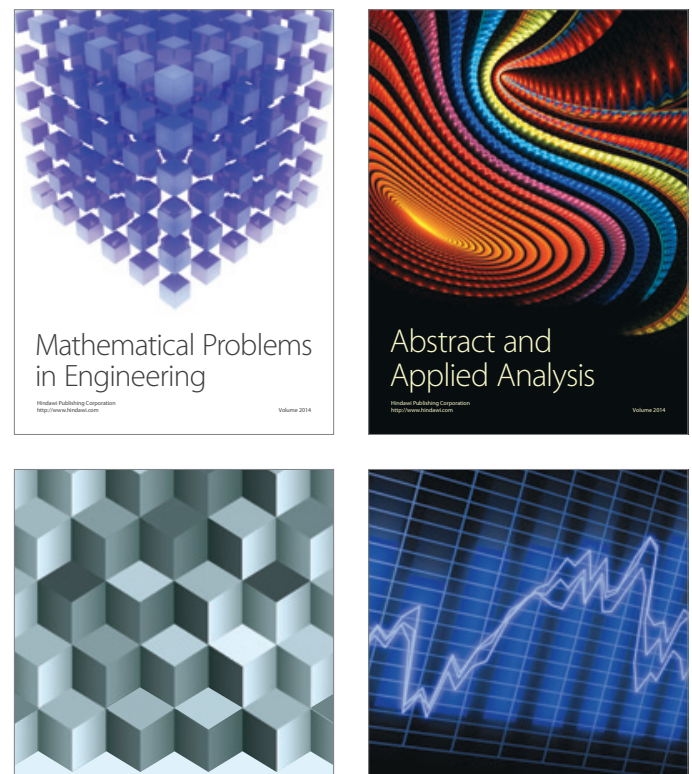

Journal of

Function Spaces

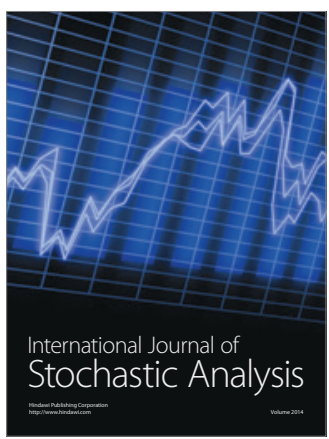

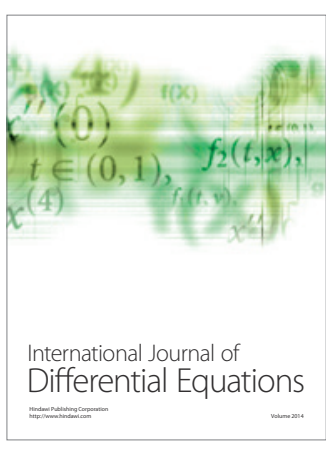
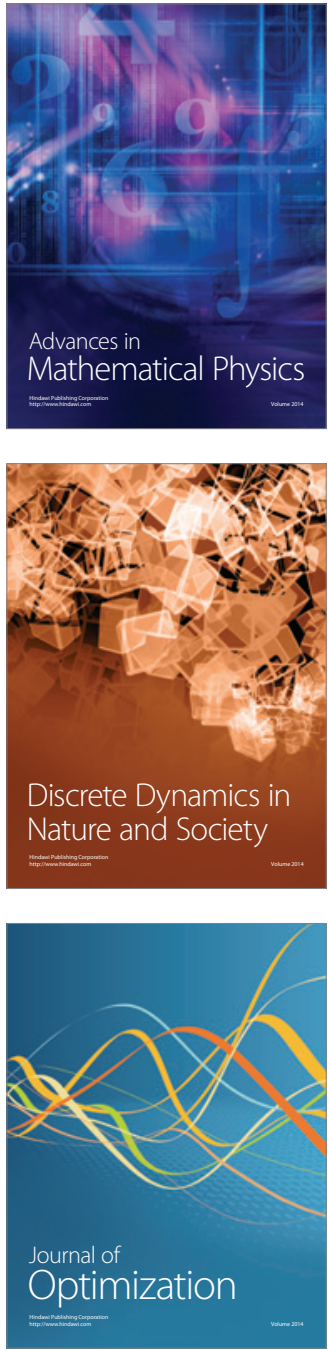